\title{
Exercise Intensity and Middle Cerebral Artery Dynamics in Humans
}

\author{
Emily Witte ${ }^{1}$, Yumei Liu ${ }^{1}$, Jaimie L. Ward ${ }^{1}$, Katie S. Kempf ${ }^{1}$, Alicen Whitaker ${ }^{1}$, Eric D. \\ Vidoni $^{2}$, Jesse C. Craig ${ }^{3}$, David C. Poole ${ }^{3}$, and Sandra A. Billinger ${ }^{1,{ }^{*}}$ \\ ${ }^{1}$ University of Kansas Medical Center, Physical Therapy and Rehabilitation Science, MS 2002, \\ 3901 Rainbow Boulevard, Kansas City, KS 66160 \\ 2University of Kansas, Alzheimer's Disease Center, KU Clinical Research Center 4350 Shawnee \\ Mission Parkway, MS 6002, Fairway, KS 66205 \\ ${ }^{3}$ Kansas State University, Departments of Kinesiology, 131 Coles Hall, 920 Denison Ave, \\ Manhattan, KS 66506
}

\begin{abstract}
Despite its necessity for understanding healthy brain aging, the influence of exercise intensity on cerebrovascular kinetics is currently unknown. We, therefore characterized middle cerebral artery blood flow velocity (MCAv) kinetics associated with two exercise intensities: low and moderate. We hypothesized that increasing exercise intensity would increase the MCAv amplitude response (Amp) and that age and estimated fitness (VंO2max) would be related to Amp. Baseline (BL) values were collected for 90 -seconds followed by a 6 -minute exercise bout. Heart rate, end-tidal $\mathrm{CO}_{2}$, mean arterial pressure and MCAv were recorded throughout. MCAv kinetics were described by Amp, time delay (TD) and time constant ( $\tau$ ). Sixty-four adults completed the study. Amp was greater during moderate compared to low exercise intensity $(\mathrm{p}<0.001)$ while no difference was observed in either TD $(\mathrm{p}=0.65)$ or $\tau(\mathrm{p}=0.47)$. Amp was negatively associated with age $(\mathrm{p}<0.01)$ and positively correlated with estimated $\mathrm{V} O 2 \mathrm{max}(\mathrm{p}<0.01)$. Although Amp declines with age, maintaining higher $\dot{\mathrm{V} O} 2 \mathrm{max}$ may benefit the cerebrovascular response to exercise.
\end{abstract}

\section{Keywords}

Cerebral blood flow velocity; Acute exercise; Aging; Aerobic fitness; Blood pressure; Middle cerebral artery

\footnotetext{
*Corresponding Author: Sandra A. Billinger, PhD, sbillinger@kumc.edu, Address: University of Kansas Medical Center, Physical Therapy and Rehabilitation Science, Mail Stop 2002, 3901 Rainbow Boulevard, Kansas City, KS 66160. Author Contribution Statement:

SB, EV, DP, JC and RM designed the study. SB, EW, YL, JW, KK, and AW contributed to the data collection. YL and EW performed the transcranial Doppler ultrasound measurements. RM and EV provided expertise for the custom written software. EW, JW, EV, and SB performed the data analysis. EW, EV and SB performed the statistical analyses. EW, JC, DP and SB wrote the manuscript. All authors commented on the manuscript and approved the final version of the manuscript.

Declarations of interest: none

Publisher's Disclaimer: This is a PDF file of an unedited manuscript that has been accepted for publication. As a service to our customers we are providing this early version of the manuscript. The manuscript will undergo copyediting, typesetting, and review of the resulting proof before it is published in its final citable form. Please note that during the production process errors may be discovered which could affect the content, and all legal disclaimers that apply to the journal pertain.
} 


\section{Introduction}

Responsive vascular control during exercise is critical to provide adequate blood flow and match $\mathrm{O}_{2}$ delivery temporally and spatially to $\mathrm{O}_{2}$ demands within and among the working muscles and other organs in the body.(Fisher et al. 2008, Ogoh et al. 2005, Heinonen et al. 2015, Koga et al. 2014) Compared to rest, maximal exercise may increase cardiac output up to $\sim 8$-fold with the majority of blood flow being distributed to the contracting skeletal muscle. (Joyner and Casey 2015) By comparison the cerebral circulation supports only a relatively modest increase in blood flow ( 0.15-0.30 fold). (Smith and Ainslie 2017) Unlike skeletal muscle where some volumetric expansion is tolerated during exercise, tight control over cerebral blood flow elevation is vital to avoid drastic changes in intracranial pressure, which could damage the blood-brain barrier. (Tzeng and Ainslie 2014) Understanding the cerebrovascular response kinetics following exercise onset across a broad range of ages and fitness levels may provide insights into the underlying control processes and their exercise intensity-dependence.

Previous work has examined the amplitude of steady state changes in mean middle cerebral artery blood flow velocity (MCAv) during various intensities of cycling exercise in young adults ( $<30$ years of age) (Ide, Horn, and Secher 1999, Ogoh et al. 2005, Fisher et al. 2008) and older adults (Fisher et al. 2008). In these studies, participants were free from cardiovascular and pulmonary disease and not taking prescribed or over the counter medications.(Fisher et al. 2008, Ogoh et al. 2005) While Fisher et al. (2008) found increases in MCAv from rest and both low and moderate exercise intensities, it was not possible to discriminate different MCAv response amplitudes between intensities. In contrast, whereas Ogoh et al. (2005) could not detect any MCAv response to low intensity exercise, there was an increase during moderate intensity exercise. The modest sample sizes ( $\mathrm{n}=7$ young, Ogoh et al. 2005; $n=9$ young and $n=10$ adults, Fisher et al. 2008) likely undermined the statistical power. Previously, our laboratory applied a novel method to assess the MCAv kinetics response from rest to moderate intensity exercise. (Billinger, Craig, et al. 2017) Similar to other physiological processes such as breathing and gas exchange (Poole and Jones 2012), cellular energetics and muscle oxygenation (Behnke et al. 2002, Behnke et al. 2001) we believe MCAv kinetics analyses could provide a more complete understanding of the fundamental mechanisms controlling cerebrovascular function with the potential to increase our knowledge of cerebrovascular health across the continuum of aging and disease processes (i.e.: stroke, vascular dementia).

In our previous work, we reported that the change $(\Delta)$ in MAP accounted for less than $40 \%$ of the variability in the MCAv Amplitude (Amp). (Billinger, Craig, et al. 2017) However, our sample size was small with 8 healthy young adults and 3 healthy older adults. A larger subject population as studied herein provides the opportunity to investigate rigorously the relationship between underlying physiologic mechanisms and the cerebrovascular response to exercise.

Whereas advancing age accounts for a decline in resting MCAv independent of exercise training in normotensive, non-obese males $(\mathrm{n}=307)$ free from overt chronic disease, MCAv is higher in endurance trained males (Ainslie et al. 2008); supporting implementation of 
chronic exercise for healthy brain aging. We recently showed that older adults $(n=14)$ have decreased baseline MCAv and a reduced MCAv Amp from rest to moderate intensity exercise when compared to their younger counterparts $(n=15)$.(Ward et al. 2018) The present investigation specifically recruited a large and heterogenous subject population with respect to sex, age and exercise training or fitness (estimated using a non-exercise based prediction of VंO2max (Billinger, Craig, et al. 2017, Mattlage et al. 2016, Mattlage et al. 2017, Billinger, Sisante, et al. 2017) to enhance ecological validity in our investigation of exercise intensity and MCAv kinetics.

The primary objective of the present investigation was to measure the MCAv kinetics response at the extremes of sub-lactate threshold metabolic rates (i.e., low and moderate intensity exercise). Heavy and severe exercise intensities (i.e. >lactate threshold) induce hyperventilation and hypocapnia that constricts the cerebral vasculature thereby reducing MCAv and were specifically avoided. (Willie et al. 2012) We tested the hypothesis that increasing work rate from low to moderate intensity would increase the MCAv Amp and possibly the speed of the MCAv kinetics response (i.e., time constant, $\tau$ ). As a secondary objective, across exercise intensities we explored the relationships between MCAv Amp and: 1) $\triangle \mathrm{MAP}, \Delta \mathrm{HR}$ and $\Delta \mathrm{P}_{\mathrm{ET}} \mathrm{CO}_{2}$ and 2) age and fitness expressed as estimated $\dot{\mathrm{V} O} 2 \mathrm{max}$.

\section{Materials and Methods}

\subsection{Study Design}

This study was completed during a single visit to the laboratory. Briefly, during the visit participants completed initial paperwork, protocol familiarization, additional paperwork, and the experimental protocol. The experimental protocol consisted of two separate 6-minute bouts of exercise at low and moderate intensity, respectively. The entire visit lasted approximately 3 hours. The outcomes and protocol are described in greater detail below.

\subsection{Participant Criteria}

Participants were screened over the phone or in person using our established inclusion/ exclusion criteria. (Billinger, Craig, et al. 2017) Inclusion criteria were: 1) 20-85 years of age, 2) ability to perform repeated bouts of exercise, and 3) ability to travel to the University of Kansas Medical Center for testing. Exclusion criteria were: 1) inability of study staff to acquire signal of the MCA using transcranial Doppler ultrasound, 2) inability to perform the alternating leg movements on the seated recumbent stepper 3) diagnosis of Parkinson's disease, mild cognitive impairment, Alzheimer's disease or multiple sclerosis, 4) pulmonary disease or dependency on supplemental oxygen or 5) diagnosis of myocardial infarction or heart failure. Before reporting to the laboratory at the University of Kansas Medical Center, participants were asked to abstain from food for two hours (Fisher et al. 2008, Kaur et al. 2018, Ogoh et al. 2005), caffeine for a minimum of six hours, (Ainslie et al. 2008) and vigorous exercise for twelve hours prior to testing. (Ogoh et al. 2005, Billinger, Craig, et al. 2017) All female participants were verbally questioned regarding menstrual status. We did not directly assess hormone level but premenopausal females exercised during the early follicular phase (Days 1-7) of the menstrual cycle. (Fisher et al. 2008, Billinger, Craig, et al. 2017, Thijssen et al. 2011)

Respir Physiol Neurobiol. Author manuscript; available in PMC 2020 April 01. 
Participants who were 60 years of age and older were recruited as part of an ongoing Alzheimer's research program at the KU Alzheimer's Disease Center. These individuals were classified as cognitively normal/non-demented based on neuropsychological testing defined as no scores greater than 1.5 standard deviations below the mean on two or more tests in the National Alzheimer's Coordinating Center Uniform Data Set and a Clinical Dementia Rating $=0$. The older individuals were determined to have no evidence of neurological disease or stroke using MRI. All older adult females were confirmed to be postmenopausal as part of their clinic visit. The University of Kansas Medical Center Human Subjects Committee approved all experimental procedures, which complied with the Declaration of Helsinki. Institutionally approved written informed consent was obtained from each individual prior to participation in the study. After written informed consent was obtained, resting heart rate (HR) was taken by a hand-held device (Tuffsat Ohmeda, GE Healthcare, Chicago, IL).

\subsection{Familiarization}

The laboratory room for the experimental session was dimly lit, quiet and temperature maintained between $22-24^{\circ} \mathrm{C}$ (Billinger, Sisante, Alqahtani, et al. 2016, Billinger, Sisante, Mattlage, et al. 2016, Billinger, Craig, et al. 2017) and external stimuli were kept to a minimum. The familiarization session was completed on the same day as the study procedure. (Billinger, Craig, et al. 2017, Pescatello and American College of Sports Medicine. 2014) Each participant was first familiarized with the experimental protocol using the seated recumbent stepper (T5XR Nustep, Ann Arbor, MI).

Throughout the familiarization, participants practiced keeping the prescribed step rate of 120 steps/min while the target work rate, which was based on HR, was determined. The target work rate was identified by setting the resistance to 40 watts and then adjusted at a rate of 10 watts every $30 \mathrm{~s}$ until their target heart rate for the exercise intensity was achieved and maintained for one minute. Participants were instructed to breathe only through their nose during the experiments. A nasal cannula was placed in the participants' nares and if needed, adjustments to the position of the nasal cannula were made to ensure optimal end-tidal carbon dioxide $\left(\mathrm{P}_{\mathrm{ET}} \mathrm{CO}_{2}\right.$ in $\left.\mathrm{mmHg}\right)$ reading.

\subsection{Additional Outcomes}

Following the familiarization session, height and weight were obtained. We then gathered information regarding participant demographics, information pertaining to past medical history, physical activity participation, American College of Sports Medicine (ACSM) cardiac risk stratification (Pescatello and American College of Sports Medicine. 2014) and a previously established and validated non-exercise estimated VंO2max questionnaire in healthy (Jurca et al. 2005) and older adults.(Mailey et al. 2010) This non-exercise prediction equation uses the following variables: sex, age, body mass index (BMI), resting heart rate, and a self-reported measure of physical activity for estimated metabolic equivalents and then multiplied by $3.5 \mathrm{~mL}^{*} \mathrm{~kg}^{-1 *} \mathrm{~min}^{-1}$ to obtain the estimated $\mathrm{V} O 2 \mathrm{max}$ value. We consider that, whereas this procedure is not the gold standard for VंO2max as measured using pulmonary gas exchange in conjunction with ramp and constant-load (validation bout) procedures (Poole and Jones 2017), it confers a broad ecological validity and can be implemented 
across non-specialist environments and laboratories. This portion lasted between 20-30 minutes. After the questionnaires, the participants returned to the laboratory room for the experimental procedure set up and sat quietly on the recumbent stepper for 20 minutes.

\subsection{Experimental Protocol}

2.5.1 Protocol Set Up-With respect to the exercise response we have previously demonstrated that there is no significant difference in the right versus left MCAv response to exercise (Billinger, Craig, et al. 2017). Herein, the left MCA was the primary vessel of interest. If the signal was not obtainable, then the right side was used. (Billinger, Craig, et al. 2017) Briefly, participants were instrumented with the following equipment in the seated position: transcranial Doppler ultrasound (TCD) (Multigon Industries Inc. Yonkers, NY), beat-to-beat mean arterial blood pressure (MAP) (Finometer, Finapres Medical Systems, Amsterdam, The Netherlands) on the left middle finger, end-tidal carbon dioxide $\left(\mathrm{P}_{\mathrm{ET}} \mathrm{CO}_{2}\right.$ in $\mathrm{mmHg}$ ) via nasal cannula (BCI Capnocheck Sleep 9004 Smiths Medical, Dublin, OH) and HR via electrocardiogram (Cardiocard, Nasiff Associates, Central Square, NY). Data was acquired through an analog-to-digital data acquisition unit (NI-USB-6212, National Instruments) and custom written software operating in MATLAB (v2014a, The Mathworks Inc. Natick, MA).

2.5.2 Experiment-The order of low and moderate intensity exercise was randomized using a random binary number generator. We used the Karvonen method to determine the appropriate HR range for low and moderate intensity. HR range $=[\%$ exercise intensity (agepredicted HR max-resting HR)] + resting HR. Using the ACSM's Guidelines for Exercise Testing and Prescription, we used the following HR ranges: 30-40\% (low) and 45-55\% (moderate). (American College of Sports et al. 2018) For each bout, participants were instructed to maintain a step rate of approximately 120 steps per minute throughout the entire exercise bout and resistance was adjusted to obtain the targeted workloads and HR range. (Billinger, Craig, et al. 2017) The baseline (BL) recording lasted 90 seconds, followed by 6 minutes of exercise at the targeted HR range. This is the standard duration utilized for determining the pulmonary gas exchange response to exercise in the low and moderate exercise intensities. (Poole and Jones 2012) After each exercise bout, participants remained in the seated position resting quietly for a minimum of 10 minutes or until all outcome measures returned to baseline values.

2.5.3 Data Acquisition-All variables were sampled at $500 \mathrm{~Hz}$ and then interpolated to $2.0 \mathrm{~Hz}$. Three-second averages were calculated and then smoothed with a $9 \mathrm{~s}$ sliding window average. (Billinger, Craig, et al. 2017) We modeled the exercise response using $\mathrm{R}$ version 3.2.4 and the nls function package (R Core team, Vienna, Austria) (Team 2013). Data with $\mathrm{R}$-to-R intervals greater than $5 \mathrm{~Hz}$ or changes in peak blood flow velocity greater than 10 $\mathrm{cm} / \mathrm{s}$ in a single cardiac cycle were considered artifact and censored. Acquisitions with more than $15 \%$ of data points censored were discarded.

\subsection{MCAv and Kinetics Response}

Based on our previous work, (Billinger, Craig, et al. 2017, Ward et al. 2018) kinetics were modeled on the MCA over the entire exercise bout with a mono-exponential model: 


$$
M C A V(t)=B L^{*}(t<=T D)+(B L+A m p(1-e-(t-T D) / \tau) *(t>T D)
$$

where $\operatorname{MCA}_{\mathrm{V}}(t)$ is the $\mathrm{MCA}_{\mathrm{V}}$ at any point in time, $\mathrm{BL}$ is the baseline before the onset of exercise, Amp is the peak amplitude of the response above resting BL, TD is the time delay proceeding the increase in $\mathrm{MCA}_{\mathrm{V}}$, and $\tau$ is the time constant or time-to- $63 \%$ of the steadystate response. Mean response time (MRT) was calculated as the sum of TD and $\tau$. Coefficients of variation for these parameters range from $3 \%$ for BL, $12 \%$ for MRT and $\tau$ and 14\% for Amp. (Billinger, Craig, et al. 2017)

\subsection{Statistical Analysis}

We performed parametric (one-way repeated measures ANOVA) and non-parametric tests (Wilcoxon) as appropriate following visual inspection of probability plots and ShapiroWilk tests. Wilcoxon tests were used for $\tau$ and MRT analyses. Pearson coefficients were used to examine the relationship between MCAv Amp with: 1) physiologic measures: $\triangle \mathrm{MAP}, \Delta \mathrm{HR}$, $\Delta \mathrm{P}_{\mathrm{ET}} \mathrm{CO}_{2}$ and 2) age and estimated $\dot{\mathrm{V}}_{2}$ max. To understand the strength of the relationship between MCAv amp and the selected measures, we used defined criteria: Pearson's coefficient $(r)=\leq 0.35$, weak relationship; $r=0.36-0.67$, moderate relationship; $r=0.68-$ 1.0, strong relationship. (Taylor 1990) Statistical significance was evaluated at $\mathrm{a}=0.05$ to protect against Type I error. Data are presented as mean \pm standard deviation unless otherwise noted. Data analysis was performed with SPSS Version 22 (IBM, Armonk, NY) for Windows

\section{Results}

Of the 73 participants enrolled, complete data sets for low and moderate exercise intensities were obtained for 64 individuals. The reasons for not including 9 subjects were: 1) missing data for either low or moderate intensity exercise $(n=5)$, and 2) poor signal acquisition during exercise for MCAv data $(n=4)$. Participants were $46.4 \pm 18.9$ years (range: 20-83 years). Participant demographics are found in Table 1. Exercise data are presented in Table 2.

\subsection{MCAv Kinetics and Exercise Intensity}

BL values for all measures were not statistically different prior to low or moderate exercise, suggesting that participants had ample rest in between the exercise bouts. BL was related to the Amp during low $(r=0.26, p=0.04)$ and moderate intensity $(r=0.47, p<0.01)$ exercise. We report that Amp was significantly increased during moderate intensity compared to low intensity ( $\mathrm{p}=0.003$ ) but MCAv $\tau$ and mean response time (MRT, TD $+\tau$ ) were not statistically different. Figure 1 and Table 2 demonstrate that the MCAv response amplitude increased systematically with the greater work rates. During steady state exercise, MAP and HR were significantly higher during moderate intensity when compared to the low intensity exercise bout. Results were not different controlling for age except for SS MAP, which was no longer significant. 


\subsection{MCAv Amp and Change in MAP, $\mathrm{HR}$ and $\mathrm{P}_{\mathrm{ET}} \mathrm{CO}_{2}$}

Low intensity exercise resulted in a weak correlation between MCAv Amp and $\triangle \mathrm{HR}$ $(\mathrm{r}=0.34, \mathrm{p}=0.006)$ but not for $\triangle \mathrm{MAP}(\mathrm{r}=0.24, \mathrm{p}=0.06)$ or $\Delta \mathrm{P}_{\mathrm{ET}} \mathrm{CO}_{2}(\mathrm{r}=0.18, \mathrm{p}=0.16)$. Moderate intensity exercise resulted in a moderate correlation between MCAv Amp and 1) $\Delta \mathrm{HR}(\mathrm{r}=0.53, \mathrm{p}<0.001)$ and 2) $\Delta \mathrm{P}_{\mathrm{ET}} \mathrm{CO}_{2}(\mathrm{r}=0.47, \mathrm{p}<0.001)$ with weak correlations between $\triangle \mathrm{MAP}(\mathrm{r}=0.25, \mathrm{p}=0.05)$.

\subsection{Age and Estimated VंO2max}

BL was weakly associated with estimated $\dot{\mathrm{V}}_{2} \max (\mathrm{r}=0.34, \mathrm{p}=0.006)$ and moderately associated with age $(\mathrm{r}=-0.55, \mathrm{p}<0.01)$. There was a moderate negative correlation between MCAv Amp and age for both low $(r=-0.40, p=0.001)$ and moderate intensity exercise $(r=$ $-0.42, \mathrm{p}=0.001$ ), Figure 2. In contrast, $\dot{\mathrm{V}}_{2}$ max moderately correlated with MCAv Amp for the low $(r=0.41, p=0.001)$ and moderate $(r=0.50, p<0.001)$ intensity exercise, Figure 3.

\section{Discussion}

These data provide novel insights into the dynamic MCAv response across the rest-exercise transition during the rapid changes in MAP and HR associated with initiating different sublactate threshold exercise intensities in adults. To our knowledge, the present investigation is the first to measure the rest-exercise transition reporting the MCAv kinetics parameters (i.e., amplitude, TD and $\tau$ ) as a function of increasing constant-load work rates. We demonstrate that increasing work rates systematically elevate MCAv Amp (Table 2, Figure 1). Interestingly, and in contrast to our hypothesis, this increased MCAv response amplitude was achieved without any significant difference in the TD and $\tau$ parameters. In this respect, the MCAv response demonstrates classic linear first order characteristics. These findings support that, if therapeutic exercise interventions seek to elicit the greatest increase in MCAv, the highest moderate intensity exercise (i.e., below the lactate or gas exchange threshold) work rate should be performed.

\subsection{MCAv Kinetics}

A strength of our experimental protocol was the randomization of low and moderate intensity exercise to avoid any practice learning or other ordering effects. These data presented here demonstrate that whether performing low (30-40\% HRR) or moderate (45$55 \%$ HRR) intensity exercise, both intensities increased MCAv Amp from BL, which may have relevance when considering exercise intensity for healthy lifestyle interventions in the general population especially those who have physical limitations (i.e.: stroke) where other exercise paradigms or intensities are not feasible. The finding that the moderate intensity exercise bout elicited a significantly greater MCAv Amp than the lower intensity counterpart is an important consideration for future work focusing on exercise intensity and brain health. (Lucas et al. 2015)

In addition to MCAv Amp, we tested the hypothesis that increasing work rate would increase the speed of the MCAv kinetics response (i.e., TD and $\tau$ ). We know that within skeletal muscle there is an immediate hyperemic response that occurs within the first contraction-relaxation cycle following the onset of exercise (Kindig, Richardson, and Poole 
2002) which serves to match $\mathrm{O}_{2}$ delivery to $\mathrm{O}_{2}$ requirements $\left(\dot{V} \mathrm{O}_{2}\right.$ ) supporting mitochondrial function and limiting the incurred $\mathrm{O}_{2}$ deficit. (Behnke et al. 2002, Poole and Jones 2012) This response is supported by a rapid arteriolar dilation that occurs within several seconds of contractions (Behnke and Delp 2010, Poole and Jones 2012) and facilitates a close-to-exponential increase in muscle blood flow. (Grassi et al. 1996) Based upon these responses in skeletal muscle we hypothesized and demonstrated that the MCAv response to exercise would conform to a time-delay+exponential model. (Billinger, Craig, et al. 2017) Our findings herein, akin to skeletal muscle blood flow and $\dot{V} \mathrm{O}_{2}$ (Whipp BJ 1980, Grassi et al. 1996, Poole and Jones 2012), but, contrary to our hypothesis, suggest that the MCAv response to exercise conforms to a linear first order process such that, for increases in work rate the amplitude increased systematically but the kinetics $(\mathrm{TD}, \tau)$ parameters are invariant. Table 2 and Figure 1 support this finding. Moreover, despite that the TD and $\tau$ are no doubt protocol-specific and work rate was increased over the course of $30 \mathrm{~s}$ by necessity to keep head movement to a minimum, the measured mean $\tau$ 's (Table 2) were not appreciably different to those for leg blood flow following the onset of cycling exercise (e.g., $34 \pm 9$ s). (Grassi et al. 1996) However, the group mean MCAv TDs modeled herein to best fit the subsequent exponential MCAv increase, were markedly slower (i.e., 43-45 s) compared to just several seconds in Grassi et al.'s investigation in exercising muscles. (Grassi et al. 1996) Although further investigation is needed, the possibility exists that the cerebrovascular system may have protective mechanisms in place to avoid such rapid responses or, quite possibly, the kinetics of increased brain metabolism could be slower than that of skeletal muscle.

\subsection{Change in MCAv, MAP, $\mathrm{HR}$ and $\mathrm{P}_{\mathrm{ET}} \mathrm{CO}_{2}$ across work rates}

As expected, all exercise work rates systematically increased MAP and HR from baseline values ( $\mathrm{p}<0.001$ ). In contrast, whereas $\mathrm{P}_{\mathrm{ET}} \mathrm{CO}_{2}$ was elevated as expected, the response was not different between work intensities (Table 2). Our proof-of-concept "Innovative Methodologies" paper (Billinger, Craig, et al. 2017) and recent publication (Ward et al. 2018) supported that older individuals evince a reduced MCAv Amp response to moderate intensity exercise. However, the present investigation purposefully did not formally stratify across age groups. Indeed, we considered that it was important to examine the ecological validity of our MCAv measurement and modeling techniques to illustrate the cerebrovascular response to increasing work rates across a broad and continuous age range (young to older adults).

In accord with our previous findings (Billinger, Craig, et al. 2017) and others (Brassard et al. 2017, Ide and Secher 2000, Fisher et al. 2008) we were unable to discern any overarching relationships between the MCAv Amp and $\triangle \mathrm{MAP}, \Delta \mathrm{P}_{\mathrm{ET}} \mathrm{CO}_{2}$, or $\triangle \mathrm{HR}$. Whereas MCAv Amp was weakly associated with our physiologic variables $\left(\triangle \mathrm{HR}, \triangle \mathrm{MAP}\right.$ and $\left.\Delta \mathrm{P}_{\mathrm{ET}} \mathrm{CO}_{2}\right)$ during low intensity exercise, during the moderate intensity work rate, the relationship between MCAv Amp with $\triangle \mathrm{HR}$ and $\Delta \mathrm{P}_{\mathrm{ET}} \mathrm{CO}_{2}$ was strengthened while the correlation between $\triangle \mathrm{MAP}$ remained unchanged from that found during the low intensity work rate. However, as $\triangle \mathrm{P}_{\mathrm{ET}} \mathrm{CO}_{2}$ changes in the absence of altered arterial $\mathrm{PCO}_{2}$, particularly at low to moderate intensities, this latter association was not considered relevant to understanding MCAv Amp herein. 
There are several potential explanations for the lack of a consistent correlation between MCAv Amp and $\triangle \mathrm{MAP}$ during exercise. For instance, the evidence for a relationship between MCAv Amp and $\triangle \mathrm{MAP}$ is largely limited to young, healthy cohorts. However, by design the present investigation comprised a more general population encompassing a substantial age range with widely varying activity levels, lifestyles and comorbidities which is further complicated by medications. However, we believe this to be a strength of the current analysis as we provide a unique perspective regarding community-dwelling adults. Moreover, whereas previous studies typically, but not always, evaluate small groups of individuals the present investigation recruited a more substantial population of 64 individuals and older adults with documented absence of brain pathology. Therefore, this more inclusive strategy does provide greater comprehensive insights regarding the relationship between MCAv Amp and $\triangle \mathrm{MAP}$ across different exercise intensities. Lastly, it is becoming apparent that MCAv Amp may be influenced by a variety of factors including age, physical activity, hypertension, and cardiac output as well as arterial $\mathrm{PCO}_{2}$. (Ainslie et al. 2008) Interactions among these factors will likely impact the relationship between MCAv Amp and $\triangle$ MAP. Future investigation into how this relationship, or lack thereof, is impacted by advancing age and patients with multiple comorbid conditions is warranted.

\subsection{Age and Estimated $\mathrm{VO}_{2} \max$}

The present investigation revealed that age and estimated $\mathrm{V} O 2 \mathrm{max}$ were, respectively, negatively (low, $r=-0.40$, mod. $r=-0.42$ ) and positively (low, $r=0.41$, mod. $r=0.50$ ) associated with MCAv Amp for both low and moderate intensity work rates. Published work from a large dataset $(n=307)$ found evidence for a progressive decline in MCAv among older men. (Ainslie et al. 2008) Intriguingly, MCAv was elevated $9 \mathrm{~cm} \cdot \mathrm{s}^{-1}$ in the endurancetrained individuals versus controls across the age range from 18 to 79 years which the authors concluded produced a "10-year reduction in MCAv equivalent age". (Ainslie et al. 2008) The data herein broadly supports and extends this previous work as regards the relationships among age, cardiovascular fitness and MCAv. However, the present findings in this regard must be interpreted with caution because of the imprecision of non-exercise VंO2max estimates compared to state-of-the art VंO2max measurements. (Poole and Jones 2017) Future work focused on the relationship between VंO2max and cerebrovascular health might usefully incorporate rigorous exercise testing.

\subsection{Experimental Considerations}

The present investigation has several strengths including inclusion of both male and female participants, an extensive sample size and age range as well as multiple exercise levels and kinetics analyses. Notwithstanding these advantages still larger sample sizes are needed to determine "normal ranges" for MCAv kinetics profiles and how this response may be related to cerebrovascular health. Our laboratory, and others, continues to recruit individuals across age ranges, fitness levels and patients with neurologic disease to expand our knowledge in this regard. Several limitations in the study design herein should be considered.

First, we are using transcranial Doppler ultrasound to measure MCAv at rest and during exercise and are not able to measure MCA diameter. The assumption of constant MCA diameter is important for $\mathrm{MCA}_{\mathrm{V}}$ to be used as a direct proxy for cerebral blood flow.

Respir Physiol Neurobiol. Author manuscript; available in PMC 2020 April 01. 
Currently, there is uncertainty surrounding whether MCA diameter changes with exercise or not. (Hoiland and Ainslie 2016, Brothers and Zhang 2016) There is some suggestion that, even if some vascular lumen diameters do change within the vascular bed this may be negligible in larger vessels such as the MCA. (Huber 1967, Giller et al. 1993) Therefore, as in our previous work (Sisante et al. 2017, Billinger, Craig, et al. 2017) and others (Fisher et al. 2008, Ide, Horn, and Secher 1999, Ide et al. 1998) we make the assumption that MCA diameter remains constant throughout the experimental procedure.

Second, we did not assess hormone status in our female cohort. In particular, estrogen's associations with lower sympathetic activation (Minson et al. 2000), higher nitric oxide synthase expression (Kleinert et al. 1998, Fadel, Zhao, and Thomas 2003) and lower blood pressure (Kim et al. 2011) may influence cerebrovascular blood flow. While we evaluated younger females during the early follicular phase of the menstrual cycle when estrogen and progesterone levels are typically low (Fisher et al. 2008), we did not directly assess hormone levels. In addition, for those females 65 years of age and older who were postmenopausal, we did not account for hormone replacement therapy. Therefore, there is a potential for differing estrogen levels among the subjects to impact the cerebrovascular response to exercise.

Third, maximal exercise testing was not performed to determine cardiorespiratory fitness rigorously. Rather we used an estimated $\dot{\mathrm{V} O} 2$ max procedure that includes self-reported physical activity as a descriptive measure of cardiorespiratory fitness. To solidify conclusions pertaining to the relationship between cardiorespiratory fitness and MCA kinetics, exercise testing is recommended. (Poole and Jones 2017)

Fourth, we did not measure $\mathrm{MCA}_{\mathrm{V}}$ for the typical duration of a moderate intensity therapeutic exercise session (i.e., $\geq 20 \mathrm{~min}$ ). As our exercise bouts were limited to $6 \mathrm{~min}$ duration we do not know how the $\mathrm{MCA}_{\mathrm{V}}$ responds to longer durations at these work rates. However, from the 6 min of $\mathrm{MCA}_{\mathrm{V}}$ recordings during exercise we may interpret the response to support that the therapeutic bandwidth for maintaining the greatest sustained $M C A_{V}$ response may be broad enough to encompass the moderate level of exercise examined herein.

Fifth, the MCAv responses demonstrated herein were measured on the recumbent stepper and may not apply directly to other exercise modes. However, recumbent stepping has the significant advantages of permitting high-quality MCAv response profiles suitable for kinetics analyses and also is amenable for use with patients and elderly populations for whom conventional cycling or treadmill exercise may not be feasible.

Finally, we assessed the relationship between MCAv Amp and 1) age and 2) estimated cardiorespiratory fitness across a wide range of individuals. In order to investigate the effects of age and cardiorespiratory fitness (estimated $\dot{\mathrm{V}} 2 \mathrm{max}$ ) on the MCAv dynamic response, future work should recruit individuals who fit specifically into 4 groups: 1) young individuals with low fitness, 2) young individuals with high fitness, 3) older adults with low fitness and 4) older adults with higher fitness. 


\section{Conclusions}

The present investigation demonstrates that MCAv and its kinetics response profile can be characterized across low and moderate work rates. The amplitude of the initial MCAv response increased systematically with work rate whereas the TD and $\tau$ kinetics parameters were invariant. These findings provide novel evidence supporting that MCAv responds to exercise with linear, first order characteristics. MCAv Amp was negatively correlated with advancing age and positively correlated with estimated cardiorespiratory fitness. These data suggest that the MCAv Amp does decline across the aging spectrum while maintaining higher cardiorespiratory fitness may benefit cerebrovascular response to exercise. No consistent relationship was demonstrated between MCAv Amp and either $\triangle \mathrm{MAP}$ or $\triangle \mathrm{HR}$.

\section{Acknowledgments}

Funding:

This was supported by the National Institutes of Health [grant numbers K01HD067318, T32HD057850, ULTR000001, P30AG035982]; the Georgia Holland Endowment Fund; the Wolhlgemuth Faculty Scholar Award.

\section{References}

Ainslie PN, Cotter JD, George KP, Lucas S, Murrell C, Shave R, Thomas KN, Williams MJ, and Atkinson G. 2008 "Elevation in cerebral blood flow velocity with aerobic fitness throughout healthy human ageing.” J Physiol 586 (16):4005-10. doi: 10.1113/jphysiol.2008.158279. [PubMed: 18635643]

American College of Sports, Medicine, Riebe Deborah, Ehrman Jonathan K., Liguori Gary, and Magal Meir. 2018 ACSM's guidelines for exercise testing and prescription.

Behnke BJ, Barstow TJ, Kindig CA, McDonough P, Musch TI, and Poole DC. 2002 "Dynamics of oxygen uptake following exercise onset in rat skeletal muscle." Respir Physiol Neurobiol 133 (3): 229-39. [PubMed: 12425970]

Behnke BJ, and Delp MD. 2010 "Aging blunts the dynamics of vasodilation in isolated skeletal muscle resistance vessels.” J Appl Physiol (1985) 108 (1):14-20. doi: 10.1152/japplphysiol.00970.2009. [PubMed: 19797684]

Behnke BJ, Kindig CA, Musch TI, Koga S, and Poole DC. 2001 "Dynamics of microvascular oxygen pressure across the rest-exercise transition in rat skeletal muscle." Respir Physiol 126 (1):53-63. [PubMed: 11311310]

Billinger SA, Sisante JV, Alqahtani AS, Pasnoor M, and Kluding PM. 2016 "Aerobic exercise improves measures of vascular health in diabetic peripheral neuropathy." Int J Neurosci 127 (1):80 85. doi: 10.3109/00207454.2016.1144056. [PubMed: 26785723]

Billinger SA, Sisante JV, Mattlage AE, Alqahtani AS, Abraham MG, Rymer MM, and Camarata PJ. 2016 "The relationship of pro-inflammatory markers to vascular endothelial function after acute stroke.” Int J Neurosci 127 (6):486-492. doi: 10.1080/00207454.2016.1198344. [PubMed: 27266959]

Billinger SA, Sisante JV, Whitaker AA, and Abraham MG. 2017 "Time Course of Flow-Mediated Dilation and Vascular Endothelial Growth Factor following Acute Stroke.” J Stroke Cerebrovasc Dis. doi: 10.1016/j.jstrokecerebrovasdis.2017.10.039.

Billinger Sandra A., Craig Jesse C., Kwapiszeski Sarah J., Sisante Jason-Flor V., Vidoni Eric D., Maletsky Rebecca, and Poole David C.. 2017 "Dynamics of middle cerebral artery blood flow velocity during moderate intensity exercise.” Journal of Applied Physiology 122 (5):1125-1133. [PubMed: 28280106]

Patrice Brassard, Ferland-Dutil Hélène, Smirl Jonathan D., Paquette Myriam, Le Blanc Olivier, Malenfant Simon, and Ainslie Philip N.. 2017 "Evidence for hysteresis in the cerebral pressure- 
flow relationship in healthy men.” American Journal of Physiology-Heart and Circulatory Physiology 312 (4):H701-H704. doi: 10.1152/ajpheart.00790.2016. [PubMed: 28130339]

Brothers RM, and Zhang R. 2016 "CrossTalk opposing view: The middle cerebral artery diameter does not change during alterations in arterial blood gases and blood pressure.” J Physiol 594 (15):40779. doi: 10.1113/JP271884. [PubMed: 27010011]

Fadel PJ, Zhao W, and Thomas GD. 2003 "Impaired vasomodulation is associated with reduced neuronal nitric oxide synthase in skeletal muscle of ovariectomized rats." J Physiol 549 (Pt 1): 243-53. doi: 10.1113/jphysiol.2003.038828. [PubMed: 12665606]

Fisher JP, Ogoh S, Young CN, Raven PB, and Fadel PJ. 2008 "Regulation of middle cerebral artery blood velocity during dynamic exercise in humans: influence of aging." J Appl Physiol (1985) 105 (1):266-73. doi: 10.1152/japplphysiol.00118.2008. [PubMed: 18467548]

Giller CA, Bowman G, Dyer H, Mootz L, and Krippner W. 1993 "Cerebral arterial diameters during changes in blood pressure and carbon dioxide during craniotomy." Neurosurgery 32 (5):737-41; [PubMed: 8492848]

Grassi B, Poole DC, Richardson RS, Knight DR, Erickson BK, and Wagner PD. 1996 "Muscle O2 uptake kinetics in humans: implications for metabolic control." J Appl Physiol (1985) 80 (3):98898. doi: 10.1152/jappl.1996.80.3.988. [PubMed: 8964765]

Heinonen I, Koga S, Kalliokoski KK, Musch TI, and Poole DC. 2015 "Heterogeneity of Muscle Blood Flow and Metabolism: Influence of Exercise, Aging, and Disease States.” Exerc Sport Sci Rev 43 (3):117-24. doi: 10.1249/jes.0000000000000044. [PubMed: 25688763]

Hoiland RL, and Ainslie PN. 2016 "CrossTalk proposal: The middle cerebral artery diameter does change during alterations in arterial blood gases and blood pressure.” J Physiol 594 (15):4073-5. doi: 10.1113/JP271981. [PubMed: 27010010]

Huber P 1967 "Angiographic evaluation of internal carotid blood flow in patients with cerebrovascular disease.” Radiol Clin Biol 36 (2):82-90. [PubMed: 6055017]

Ide K, Horn A, and Secher NH. 1999 "Cerebral metabolic response to submaximal exercise." J Appl Physiol (1985) 87 (5):1604-8. [PubMed: 10562597]

Ide K, Pott F, Van Lieshout JJ, and Secher NH. 1998 "Middle cerebral artery blood velocity depends on cardiac output during exercise with a large muscle mass." Acta Physiol Scand 162 (1):13-20. doi: 10.1046/j.1365-201X.1998.0280f.x. [PubMed: 9492897]

Ide K, and Secher NH. 2000 "Cerebral blood flow and metabolism during exercise." Prog Neurobiol 61 (4):397-414. [PubMed: 10727781]

Joyner MJ, and Casey DP. 2015 "Regulation of increased blood flow (hyperemia) to muscles during exercise: a hierarchy of competing physiological needs.” Physiological reviews 95 (2):549-601. [PubMed: 25834232]

Jurca R, Jackson AS, LaMonte MJ, Morrow JR, Jr., Blair SN, Wareham NJ, Haskell WL, van Mechelen W, Church TS, Jakicic JM, and Laukkanen R. 2005 "Assessing cardiorespiratory fitness without performing exercise testing." Am J Prev Med 29 (3):185-93. doi: 10.1016/j.amepre. 2005.06.004.

Kaur J, Vranish JR, Barbosa TC, Washio T, Young BE, Stephens BY, Brothers RM, Ogoh S, and Fadel PJ. 2018 "Regulation of Regional Cerebral Blood Flow During Graded Reflex-Mediated Sympathetic Activation via Lower Body Negative Pressure.” J Appl Physiol (1985). doi: 10.1152/ japplphysiol.00623.2018.

Kim A, Deo SH, Vianna LC, Balanos GM, Hartwich D, Fisher JP, and Fadel PJ. 2011 "Sex differences in carotid baroreflex control of arterial blood pressure in humans: relative contribution of cardiac output and total vascular conductance.” Am J Physiol Heart Circ Physiol 301 (6):H2454-65. doi: 10.1152/ajpheart.00772.2011. [PubMed: 21963834]

Kindig CA, Richardson TE, and Poole DC. 2002 "Skeletal muscle capillary hemodynamics from rest to contractions: implications for oxygen transfer." J Appl Physiol (1985) 92 (6):2513-20. doi: 10.1152/japplphysiol.01222.2001. [PubMed: 12015367]

Kleinert H, Wallerath T, Euchenhofer C, Ihrig-Biedert I, Li H, and Forstermann U. 1998 "Estrogens increase transcription of the human endothelial NO synthase gene - Analysis of the transcription factors involved." Hypertension 31 (2):582-588. 10.1161/01.Hyp.31.2.582. [PubMed: 9461225] 
Koga S, Rossiter HB, Heinonen I, Musch TI, and Poole DC. 2014 "Dynamic heterogeneity of exercising muscle blood flow and O2 utilization." Med Sci Sports Exerc 46 (5):860-76. doi: 10.1249/MSS.0000000000000178. [PubMed: 24091989]

Lucas Samuel JE, Cotter James D, Brassard Patrice, and Bailey Damian M. 2015 "High-Intensity Interval Exercise and Cerebrovascular Health: Curiosity, Cause, and Consequence.” Journal of Cerebral Blood Flow \& Metabolism 35 (6):902-911. doi: 10.1038/jcbfm.2015.49. [PubMed: 25833341]

Mailey EL, White SM, Wojcicki TR, Szabo AN, Kramer AF, and McAuley E. 2010 "Construct validation of a non-exercise measure of cardiorespiratory fitness in older adults." BMC public health 10:59. doi: 10.1186/1471-2458-10-59. [PubMed: 20144197]

Mattlage AE, Redlin SA, Rosterman LR, Harn N, Sisante JV, Abraham MG, and Billinger SA. 2016 "Use of a Nonexercise Estimate for Prestroke Peak Vo2 During the Acute Stroke Hospital Stay." Cardiopulm Phys Ther J 27 (3):96-103. [PubMed: 27478424]

Mattlage AE, Rippee MA, Abraham MG, Sandt J, and Billinger SA. 2017 "Estimated Prestroke Peak VO2 Is Related to Circulating IGF-1 Levels During Acute Stroke.” Neurorehabil Neural Repair 31 (1):65-71. doi: 10.1177/1545968316656056. [PubMed: 27377914]

Minson CT, Halliwill JR, Young TM, and Joyner MJ. 2000 "Influence of the menstrual cycle on sympathetic activity, baroreflex sensitivity, and vascular transduction in young women." Circulation 101 (8):862-8. [PubMed: 10694525]

Ogoh S, Fadel PJ, Zhang R, Selmer C, Jans O, Secher NH, and Raven PB. 2005 "Middle cerebral artery flow velocity and pulse pressure during dynamic exercise in humans." Am J Physiol Heart Circ Physiol 288 (4):H1526-31. doi: 10.1152/ajpheart.00979.2004. [PubMed: 15591094]

Pescatello Linda S., and American College of Sports Medicine. 2014 ACSM's guidelines for exercise testing and prescription. 9th ed. Philadelphia: Wolters Kluwer/Lippincott Williams \& Wilkins Health.

Poole DC, and Jones AM. 2012 “Oxygen uptake kinetics.” Compr Physiol 2 (2):933-96. doi: 10.1002/ cphy.c100072. [PubMed: 23798293]

Poole DC, and Jones AM. 2017 "Measurement of the maximum oxygen uptake VO2max : Vo2peak is no longer acceptable." J Appl Physiol (1985) 122 (4):997-1002. doi: 10.1152/japplphysiol. 01063.2016. [PubMed: 28153947]

Sisante JV, Vidoni ED, Kirkendoll K, Ward J, Liu Y, Kwapiszeski S, Maletsky R, Burns JM, and Billinger SA. 2017 "Blunted cerebrovascular response is associated with elevated beta-amyloid." $J$ Cereb Blood Flow Metab:271678×17732449. doi: 10.1177/0271678×17732449.

Smith Kurt J., and Ainslie Philip N.. 2017 "Regulation of cerebral blood flow and metabolism during exercise.” Experimental Physiology 102 (11):1356-1371. doi: 10.1113/EP086249. [PubMed: 28786150]

Taylor Richard. 1990 "Interpretation of the correlation coefficient: a basic review." Journal of diagnostic medical sonography 6 (1):35-39.

A language and environment for statistical computing. R Foundation for Statistical Computing, Vienna, Austria.

Thijssen DH, Black MA, Pyke KE, Padilla J, Atkinson G, Harris RA, Parker B, Widlansky ME, Tschakovsky ME, and Green DJ. 2011 "Assessment of flow-mediated dilation in humans: a methodological and physiological guideline.” Am J Physiol Heart Circ Physiol 300 (1):H2-12. doi: 10.1152/ajpheart.00471.2010. [PubMed: 20952670]

Tzeng Yu-Chieh, and Ainslie Philip N.. 2014 "Blood pressure regulation IX: cerebral autoregulation under blood pressure challenges.” European Journal of Applied Physiology 114 (3):545-559. doi: 10.1007/s00421-013-2667-y. [PubMed: 23737006]

Ward JL, Craig JC, Liu Y, Vidoni ED, Malesky R, Poole DC, and Billinger SA. 2018 "Effect of Healthy Aging and Sex on Middle Cerebral Artery Blood Velocity Dynamics During Moderate Intensity Exercise.” Am J Physiol Heart Circ Physiol. doi: 10.1152/ajpheart.00129.2018.

Whipp BJ, Mahler M. 1980 "Dynamics of Pulmonary Gas Exchange during Exercise" In Pulmonary Gas Exchange, edited by John B. West, 33-96. New York, NY: Academic Press Inc.

Willie CK, Macleod DB, Shaw AD, Smith KJ, Tzeng YC, Eves ND, Ikeda K, Graham J, Lewis NC, Day TA, and Ainslie PN. 2012 "Regional brain blood flow in man during acute changes in arterial 
blood gases.” J Physiol 590 (14):3261-75. doi: 10.1113/jphysiol.2012.228551. [PubMed: 22495584] 


\section{Highlights:}

- $\quad$ Stimulus-response profiles during exercise are important for understanding control processes

- Middle cerebral artery blood flow velocity increased systematically with work rate

- $\quad$ Amplitude response associated with age and estimated fitness

- $\quad$ Maintaining fitness may benefit cerebrovascular response across aging 


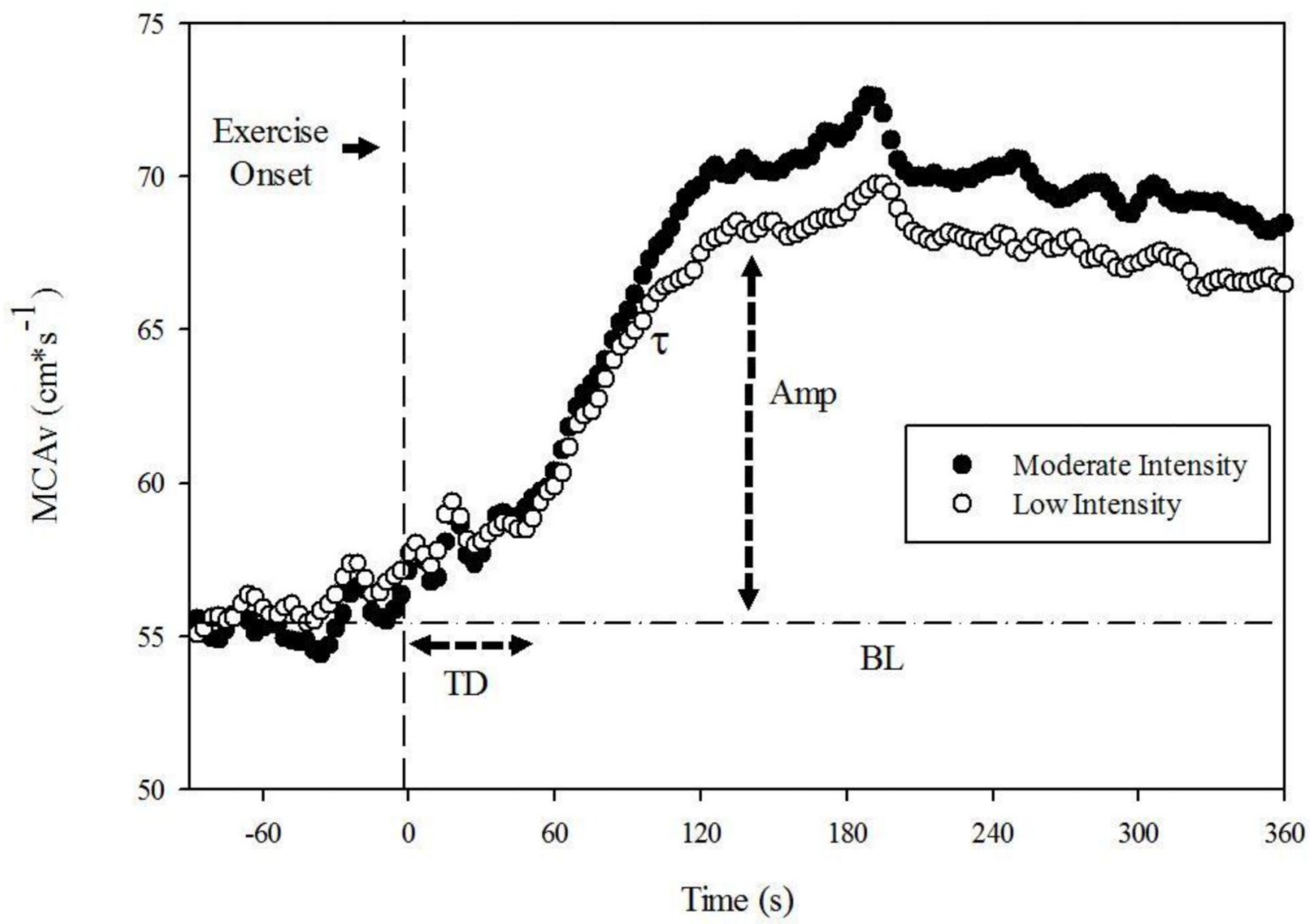

Figure 1.

Mean dynamic middle cerebral artery velocity (MCAv) responses for all participants $(n=64)$ from rest to exercise during a bout of low and moderate intensity exercise (standard errors are omitted for clarity). Time point zero indicates the onset of exercise. Key parameters are indicated: BL, Baseline; TD, Time Delay; $\tau$, Time Constant which denotes time-to- $63 \%$ of primary response (i.e., Amp, Amplitude of primary response). Please see text for further details. 


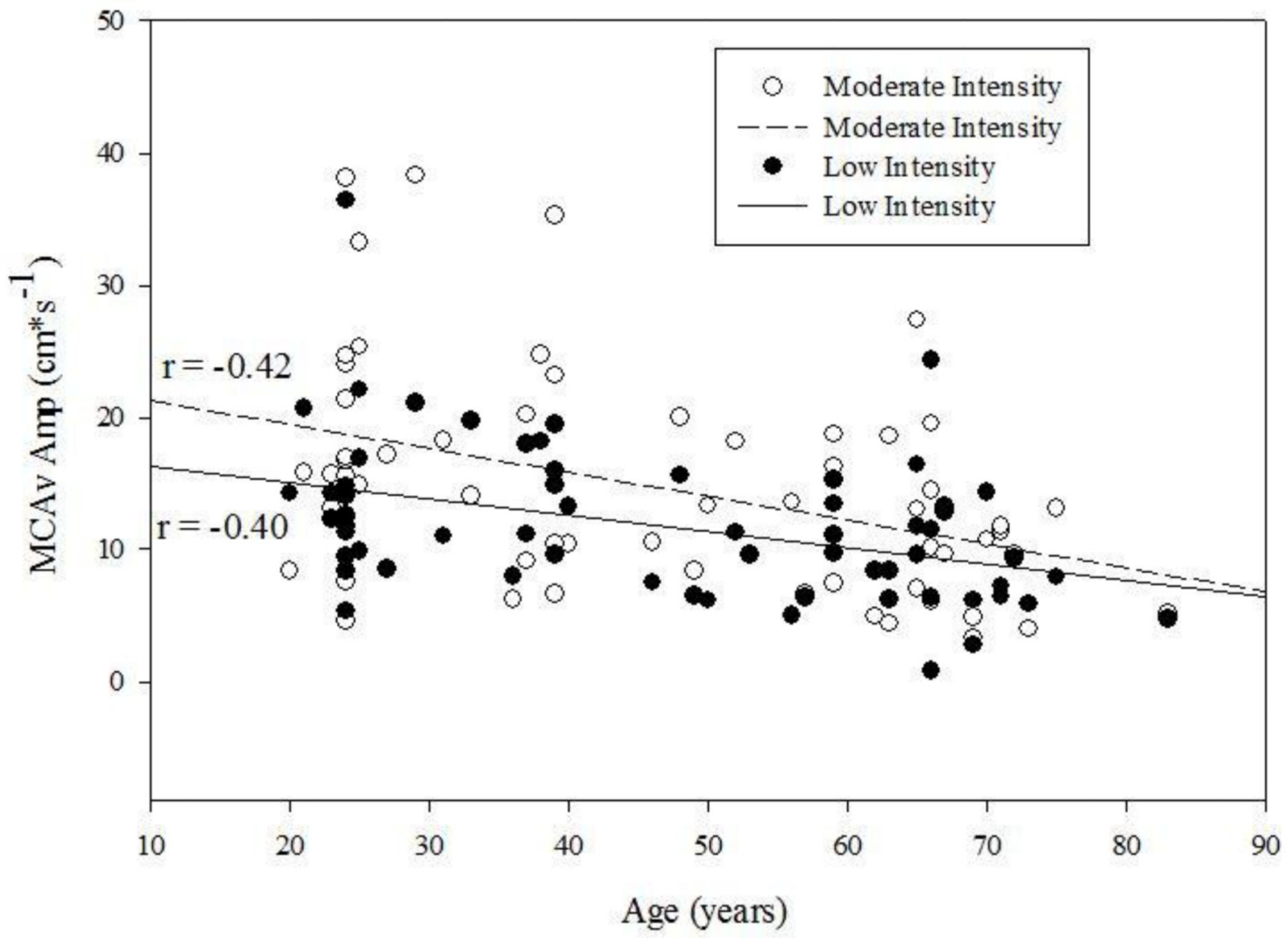

Figure 2.

Correlation between age and MCAv Amp during low $(r=-0.40, \mathrm{p}=0.001$, solid line $)$ and moderate intensity exercise $(r=-0.42, p=0.001$, dashed line). 


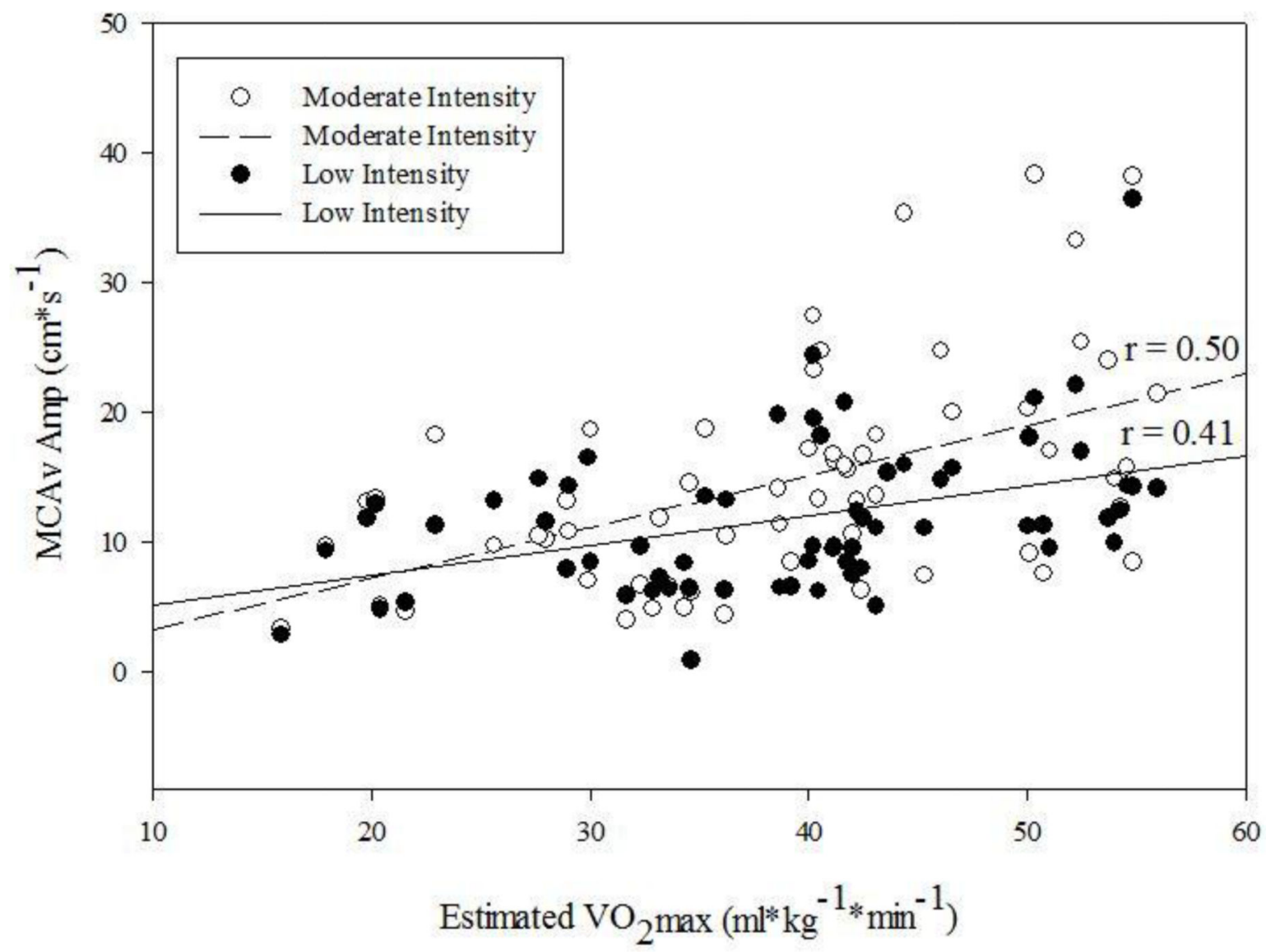

Figure 3.

$\dot{V} O 2 \max$ correlated with MCAv Amp for the low $(\mathrm{r}=0.41, \mathrm{p}=0.001$, solid line $)$ and moderate $(r=0.50, p<0.001$, dashed line) intensity exercise. 
Table 1:

Participant Demographics

\begin{tabular}{|l|c|}
\hline & Total (n=64) \\
\hline Age & $46.4 \pm 18.9$ \\
\hline BMI $\left(\mathrm{kg} \cdot \mathrm{m}^{-2}\right)$ & $23.9 \pm 4.2$ \\
\hline Estimated $\dot{V} \mathrm{O}_{2} \mathrm{max}\left(\mathrm{ml} \cdot \mathrm{kg}^{-1} \cdot \mathrm{min}^{-1}\right)$ & $39.1 \pm 10.3$ \\
\hline Male, $\mathrm{n}(\%)$ & $36(56.3)$ \\
\hline Race, $\mathrm{n}(\%)$ & \\
\hline Caucasian & $53(82.8)$ \\
\hline African American & $2(3.1)$ \\
\hline Asian & $9(14.1)$ \\
\hline Ethnicity, n (\%) & \\
\hline Hispanic & $4(6.3)$ \\
\hline Non-Hispanic & $60(93.7)$ \\
\hline CVD Risk (H:M:L), $\mathrm{n}$ & $14: 12: 38$ \\
\hline
\end{tabular}

Data are presented as mean \pm standard deviation unless otherwise indicated. Body mass index, BMI; Cardiovascular disease, CVD; High: Moderate: Low, H:M:L. 
Table 2:

Baseline and Exercise Response during Low and Moderate Intensity

\begin{tabular}{|c|c|c|c|}
\hline$n=64$ & Low & Moderate & p value \\
\hline $\operatorname{BL} \operatorname{MCAv}\left(\mathrm{cm} \cdot \mathrm{s}^{-1}\right)$ & $56.4 \pm 11.1$ & $55.7 \pm 11.3$ & 0.16 \\
\hline Amplitude $\left(\mathrm{cm} \cdot \mathrm{s}^{-1}\right)$ & $11.8 \pm 5.8$ & $14.7 \pm 8.2$ & $<0.001$ \\
\hline SS MCAv $\left(\mathrm{cm} \cdot \mathrm{s}^{-1}\right)$ & $68.2 \pm 13.8^{*}$ & $70.3 \pm 16.7^{*}$ & 0.004 \\
\hline Time Delay (s) & $43.5 \pm 33.9$ & $45.8 \pm 33.3$ & 0.65 \\
\hline$\tau(\mathrm{s})$ & $35.2 \pm 34.2$ & $31.4 \pm 25.0$ & 0.74 \\
\hline MRT (s) & $78.7 \pm 36.7$ & $77.2 \pm 38.0$ & 0.76 \\
\hline BL MAP (mmHg) & $76.5 \pm 10.9$ & $76.8 \pm 10.3$ & 0.82 \\
\hline SS MAP (mmHg) & $96.3 \pm 14.0^{*}$ & $102.6 \pm 15.5^{*}$ & $<0.001$ \\
\hline$\Delta \mathrm{MAP}(\mathrm{mmHg})$ & $19.8 \pm 9.0$ & $25.8 \pm 10.1$ & $<0.001$ \\
\hline $\mathrm{BL} \mathrm{P}_{\mathrm{ET}} \mathrm{CO}_{2}(\mathrm{mmHg})$ & $34.2 \pm 4.0$ & $34.0 \pm 3.7$ & 0.62 \\
\hline $\mathrm{SS} \mathrm{P}_{\mathrm{ET}} \mathrm{CO}_{2}(\mathrm{mmHg})$ & $40.9 \pm 4.9^{*}$ & $41.5 \pm 4.4^{*}$ & 0.05 \\
\hline$\Delta \mathrm{P}_{\mathrm{ET}} \mathrm{CO} 2(\mathrm{mmHg})$ & $6.7 \pm 3.3$ & $7.5 \pm 4.1$ & 0.05 \\
\hline BL HR (bpm) & $69.5 \pm 11.3$ & $70.9 \pm 12.4$ & 0.10 \\
\hline SS HR (bpm) & $105.8 \pm 13.3^{*}$ & $116.9 \pm 15.6^{*}$ & $<0.001$ \\
\hline$\Delta \mathrm{HR}(\mathrm{bpm})$ & $36.3 \pm 9.7$ & $46.0 \pm 11.1$ & $<0.001$ \\
\hline $\mathrm{CVRi}\left(\mathrm{mmHg} \cdot \mathrm{cm}^{-1} \cdot \mathrm{s}^{-1}\right)$ & $1.4 \pm 0.3$ & $1.4 \pm 0.3$ & 0.15 \\
\hline SS CVRi $\left(\mathrm{mmHg} \cdot \mathrm{cm}^{-1} \cdot \mathrm{s}^{-1}\right)$ & $1.5 \pm 0.4^{*}$ & $1.5 \pm 0.4^{*}$ & 0.003 \\
\hline$\Delta \mathrm{CVRi}\left(\mathrm{mmHg} \cdot \mathrm{cm}^{-1} \cdot \mathrm{s}^{-1}\right)$ & $0.06 \pm 0.2$ & $0.13 \pm 0.2$ & 0.002 \\
\hline Target Workload (Watts) & $91.7 \pm 34.4$ & $115.2 \pm 34.8$ & $<0.001$ \\
\hline
\end{tabular}

Values are presented as mean \pm standard deviation. BL, baseline; MCAv, middle cerebral artery velocity; SS, steady state; $\tau$, time constant; MRT, mean response time; MAP, mean arterial blood pressure; $\Delta$, change; $\mathrm{PETCO}_{2}$, end-tidal partial pressure of carbon dioxide; HR, heart rate; $\mathrm{CVRi}$, cerebrovascular resistance index.

values significantly different from baseline values, $\mathrm{p}<0.01$. 